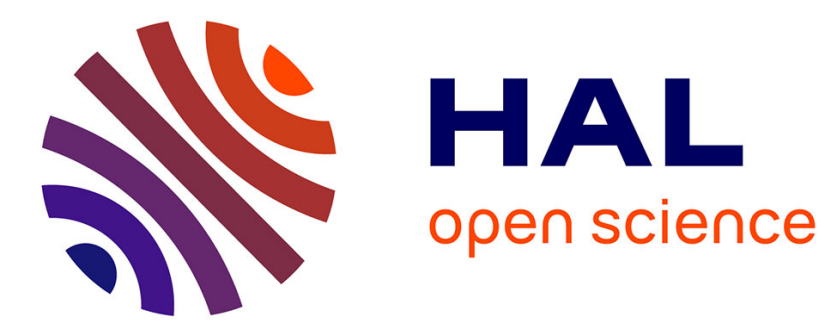

\title{
Evolution holocène de la Tunisie saharienne et présaharienne
}

Jean-Louis Ballais

\section{To cite this version:}

Jean-Louis Ballais. Evolution holocène de la Tunisie saharienne et présaharienne. Méditerranée: revue géographique des pays méditerranéens, 1991, 74 (4), pp.31-38. 10.3406/medit.1991.2735 . hal01562627

\section{HAL Id: hal-01562627 https://hal-amu.archives-ouvertes.fr/hal-01562627}

Submitted on 20 Jul 2017

HAL is a multi-disciplinary open access archive for the deposit and dissemination of scientific research documents, whether they are published or not. The documents may come from teaching and research institutions in France or abroad, or from public or private research centers.
L'archive ouverte pluridisciplinaire HAL, est destinée au dépôt et à la diffusion de documents scientifiques de niveau recherche, publiés ou non, émanant des établissements d'enseignement et de recherche français ou étrangers, des laboratoires publics ou privés. 


\section{Évolution holocène de la Tunisie saharienne et présaharienne}

In: Méditerranée, Tome 74, 4-1991. pp. 31-38.

\section{Résumé}

On peut diviser l'Holocène de la Tunisie saharienne et présaharienne en quatre sous-périodes. L'Holocène inférieur (10 000 B.P. à environ 7000 B.P.) est caractérisé par la croissance de l'humidité (fixation des dunes, réapparition de lacs, accumulation de la basse terrasse et élaboration du glacis holocène). II se termine peut-être par une courte phase sèche vers 7500 - 7000 B.P. L'Holocène moyen ( 7000 - 3500 B.P.?) est probablement la sous-période de l'optimum climatique (fin de l'accumulation de la basse terrasse, épanouissement de la pédogenèse et de l'hydrographie). A l'Holocène supérieur préhistorique commence l'assèchement climatique progressif (entaille de la basse terrasse, développement de la déflation). L'Holocène supérieur historique, après une possible courte phase humide vers 2400 B.P., se caractérise par l'accumulation de la très basse terrasse, puis son entaille et enfin par la désertification qui remobilise les sables éoliens.

\section{Abstract}

Tunisian saharian and presaharian Holocene period can be divided into four subperiods. Lower Holocene (10 000 B.P. to about 7 000 B.P.) is caracterized by increasing humidity (stabilization of dunes, reappearance of lakes, accumulation of lower terrace and genesis of Holocène glacis). A short dry phase probably took place between 7500 and 7000 B.P. Middle Holocene (7 $000-3$ 500 B.P. ?) is probably the climatic optimum subperiod (end of lower terrace building up, pedogenesis developping and evidences of good hydrologie conditions). A progressive climatic aridification began during prehistoric upper Holocene (cuttingdown of lower terrace and deflation). A probable short humid phase about 2400 B.P. marked the beginning of historic upper Holocene that is caracterized by building up then cutting-down of very low terrace and, last, by mobilization of aeolian sands due to desertification.

Citer ce document / Cite this document :

Ballais Jean-Louis. Évolution holocène de la Tunisie saharienne et présaharienne. In: Méditerranée, Tome 74, 4-1991. pp. 3138.

doi : 10.3406/medit.1991.2735

http://www.persee.fr/web/revues/home/prescript/article/medit_0025-8296_1991_num_74_4_2735 


\title{
Évolution holocène de la Tunisie saharienne et présaharienne
}

Jean-Louis BALLAIS*

Résumé - On peut diviser l'Holocène de la Tunisie saharienne et présaharienne en quatre sous-périodes. L'Holocène inférieur (10000 B.P. à environ 7000 B.P.) est caractérisé par la croissance de l'humidité (fixation des dunes, réapparition de lacs, accumulation de la basse terrasse et élaboration du glacis holocène). Il se termine peut-être par une courte phase sèche vers 7500 - 7000 B.P. L'Holocène moyen ( 7000 - 3500 B.P.?) est probablement la sous-période de l'optimum climatique (fin de l'accumulation de la basse terrasse, épanouissement de la pédogenèse et de l'hydrographie). A l'Holocène supérieur préhistorique commence l'assèchement climatique progressif (entaille de la basse terrasse, développement de la déflation). L'Holocène supérieur historique, après une possible courte phase humide vers 2400 B.P., se caractérise par l'accumulation de la très basse terrasse, puis son entaille et enfin par la désertification qui remobilise les sables éoliens.

\begin{abstract}
Tunisian saharian and presaharian Holocene period can be divided into four subperiods. Lower Holocene (10 000 B.P. to about 7000 B.P.) is caracterized by increasing humidity (stabilization of dunes, reappearance of lakes, accumulation of lower terrace and genesis of Holocene glacis). A short dry phase probably took place between 7500 and 7000 B.P. Middle Holocene (7 000 - 3500 B.P. ?) is probably the climatic optimum subperiod (end of lower terrace building up, pedogenesis developping and evidences of good hydrologic conditions). A progressive climatic aridification began during prehistoric upper Holocene (cutting-down of lower terrace and deflation). A probable short humid phase about 2400 B.P. marked the beginning of historic upper Holocene that is caracterized by building up then cutting-down of very low terrace and, last, by mobilization of aeolian sands due to desertification.
\end{abstract}

Sous réserve de confirmation par de nouvelles analyses géochimiques, minéralogiques et palyno-

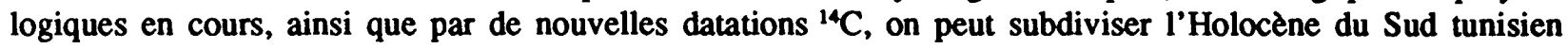
en quatre périodes: l'Holocène inférieur, l'Holocène moyen, l'Holocène supérieur préhistorique et l'Holocène supérieur historique.

L'Holocène succède à un Tardiglaciaire au cours duquel deux pulsations humides, contemporaines du Bolling et de l'Allerod, annoncent la forte humidité de l'Holocène inférieur et moyen (BALLAIS, HEDDOUCHE, 1991). Après cet optimum, le climat tend à s'aridifier jusqu'au niveau actuel, probablement atteint, pour l'essentiel, au début de la période historique.

\section{1 - L'HOLOCÉNE INFÉRIEUR (10 000 B.P. À ENVIRON 7000 B.P.): LA CROISSANCE DE L'HUMIDITÉ}

\subsection{La fixation des dunes et leur colonisation par les Capsiens}

La fixation des dunes commence, en fait, à l'extrême fin du Pléistocène supérieur, au moins sur le littoral du golfe de Gabès (fig. 1). Là, dans la station A (Capsien de faciès méridional, GRAGUEB, 1983), juste au nord de l'oued el Akarit, deux datations ${ }^{14} \mathrm{C}$ sur coquilles d'cufs d'autruche viennent d'être obtenues: $10510 \pm 349$ B.P. et $10013 \pm 281$ B.P.

Sur les sables éoliens de Bir Oum Ali (BALLAIS, BEN OUEZDOU, 1991) (fig. 1), deux escargotières capsiennes s'installent dont une datée ${ }^{14} \mathrm{C} 8260 \pm 180$ B.P. sur coquilles d'escargots (HARBI-RIAHI, 1989).

* Professeur, UFR des Sciences Géographiques et de l'Aménagement, Université d'Aix-Marseille II. 


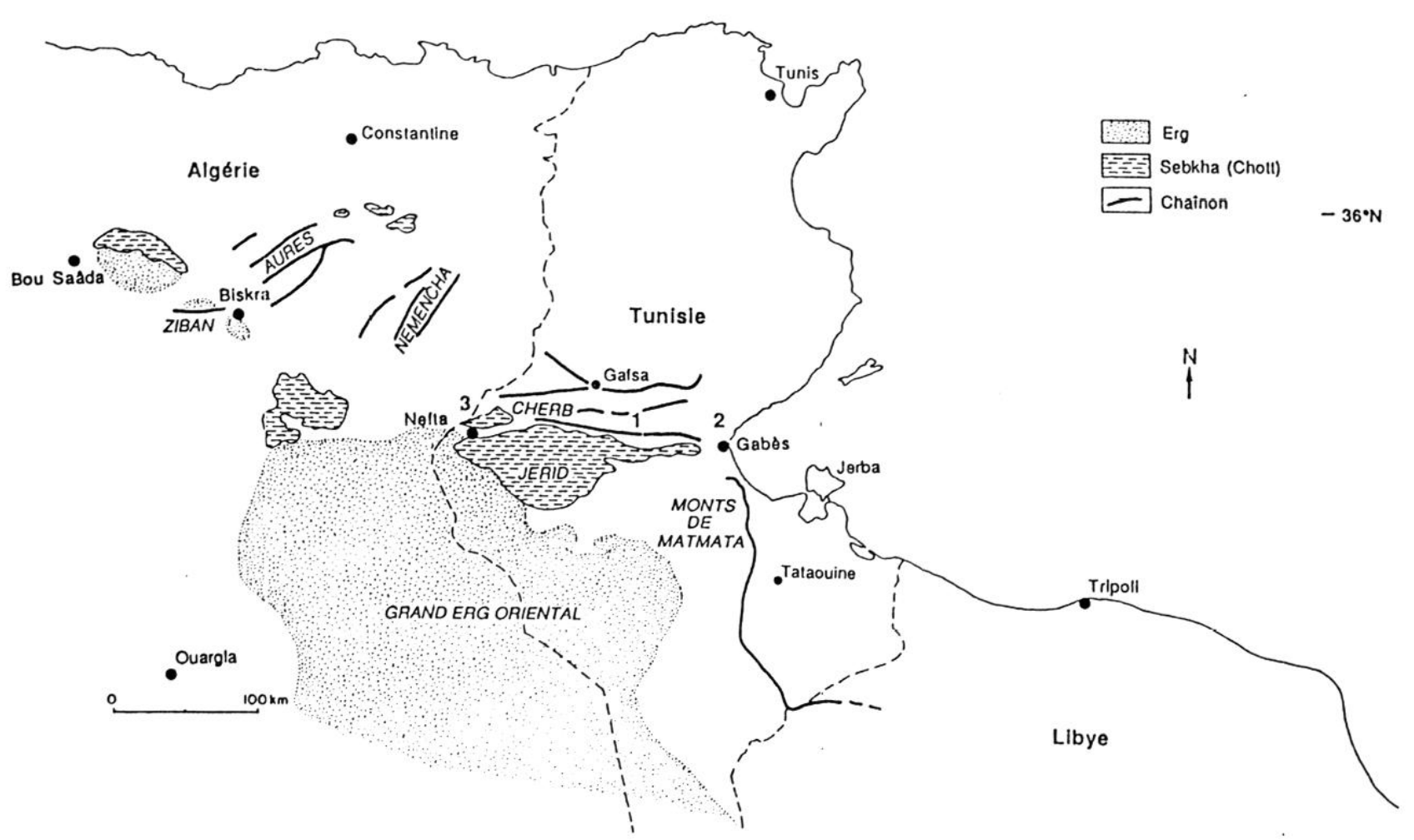

Fig. 1 - CARTE DE LOCALISATION. 1: Bir Oum Ali, 2: Oued el Akarit, 3: Erg el Oussif.

Ces Capsiens consomment alors presque exclusivement Leucochroa candidissima, principale espèce encore vivante aujourd'hui.

Enfin, sur les piémonts nord et sud du djebel Ben Younès, à l'ouest de Gafsa (fig. 1), des escargotières $s^{\prime}$ installent aussi sur des sables éliens (BEN OUEZDOU, sous presse). Bien qu'on ne puisse exclure totalement une mobilité des sables éoliens, ces installations tendent à prouver que les sables sont plus ou moins fixés à ce moment, alors qu'ils se déplaçaient encore vers 14000 B.P. (BALLAIS, HEDDOUCHE, 1991). L'augmentation des précipitations est alors bien commencée, mais leur total ne semble pas encore supérieur à l'actuel, dans le Présahara (fig. 2).

\subsection{La réapparition de lacs}

Très récemment, et pour la première fois, des paléolacs holocènes viennent d'être identifiés dans le Sud tunisien (PETIT-MAIRE et al., 1991), en particulier à Ez Zobbas, près de Larich (fig. 3). Là, vivaient des Cerastoderma glaucum datés de $8230 \pm 70$ B.P.

Cette découverte s'inscrit bien dans la logique des nombreux lacs holocènes à C. glaucum du Sahara, bien que le lac d'Ez Zobbas ait environ 1000 ans de moins que le plus proche lac, $100 \mathrm{~km}$ plus au sud, en Libye (PETIT-MAIRE, DELIBRIAS, 1979). Cela rend encore plus intrigante l'absence de lac holocène dans les Grands Chotts tunisiens. Dans l'état actuel des recherches, on n'y remarque qu'une reprise d'activité des sources prouvée par des faunules de Melanoïdes, à Toumbar et à Aîn el Atrouss, datées ${ }^{14} \mathrm{C}$ entre 9000 et 8 500B.P. après correction (ZOUARI, 1988, p. 218). Faut-il envisager, à l'Holocène inférieur, sinon un renversement de la zonation actuelle, du moins un effacement ? Ou ne s'agit-il que d'un décalage entre la réalimentation des nappes profondes et les précipitations locales? Dans ce cas, l'optimum hydrologique serait antérieur à l'optimum climatique.

\subsection{L'accumulation de la basse terrasse et l'élaboration du glacls holocène}

Il y a près de 30 ans, R. COQUE (1962) avait proposé de placer l'élaboration du glacis et l'accumulation de la basse terrasse dans l'Holocène inférieur (environ 8400 B.P. à environ 7400 B.P.), 
contemporain des Capsiens. Tout récemment, des confirmations ont été apportées. Sur l'oued el Akarit, la période qui va de 9100 à 7690 B.P. serait marquée par de forts débits qui permettent l'accumulation de la terrasse holocène $=$ formation Akarit II (ZOUARI, 1988, p. 229-230).

Au sud de Tataouine (fig. 1), sur l'oued Bir el Amir, la partie moyenne de la terrasse holocène vient d'être datée de $7026 \pm 175$ B.P., sur coquilles d'Eobania vermiculata, espèce adaptée à des pluviosités plus importantes que les moins de $100 \mathrm{~mm}$ qui tombent actuellement.

Enfin, dans l'Extrême-Sud, la partie supérieure de la terrasse de l'oued Jenain est datée de $7890 \pm 90$ B.P. et celle des oueds Makhrerouga et Abdallah de $8010 \pm 160$ B.P. Là où il tombe environ $50 \mathrm{~mm} / \mathrm{an}$, dans des flaques d'eau douce vivaient alors des Gastéropodes aquatiques, en particulier Lymnaea

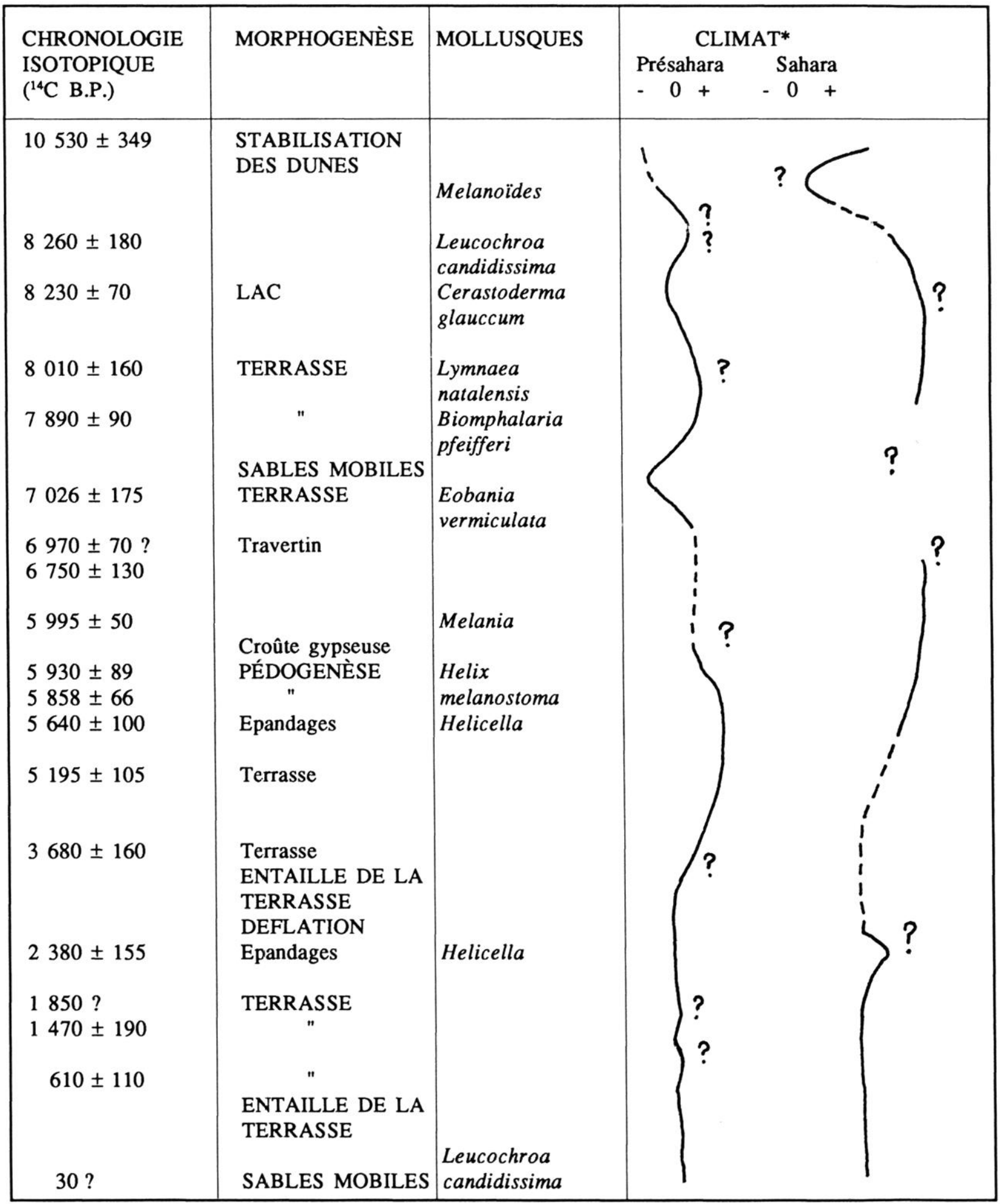

Fig. 2 - ÉVOLUTION MORPHOCLIMATIQUE HOLOCÈNE.

*humidité par rapport à l'Actuel. 
natalensis et Biomphalaria pfeifferi (PETIT-MAIRE et al., 1991). La vitesse moyenne d'accumulation de cette terrasse est de l'ordre de 1,4 mm/an (BALLAIS, 1991b).

L'extension du glacis holocène (glacis 1) a êté étendue également aux versants aspergés (BALLAIS, BEN OUEZDOU, 1991). En effet, ce glacis tronque très généralement la troisième génération de ces accumulations de sables éoliens, entre Gafsa et la chaîne du Cherb (fig. 1). A Bir Oum Ali, il est jalonné par une escargotière capsienne et, dans le djebel Atra, de nombreux sites néolithiques sont posés dessus. Enfin, à Smila, au nord-est de Tataouine, il est antérieur à un Néolithique précoce (7 $636 \pm 136$ B.P.).

Globalement donc, les écoulements sont redevenus très vigoureux, efficaces, parfois dès avant 10000 B.P. (BALLAIS, HEDDOUCHE, 1991). Si cela ne peut s'interpréter, par rapport à la forte aridité du Pléniglaciaire, que comme dû à une augmentation des précipitations locales (fig. 2), ces dernières ont dû être assez intenses pour permettre un ruissellement sur des sables dunaires récemment fixés. $L$ 'augmentation générale de la pluviosité est corroborée par les analyses palynologiques (BRUN, 1989).

\subsection{Une phase seche vers 7500 - 7000 B.P.?}

Après 7690 B.P., les débits de l'oued el Akarit diminuent (ZOUARI, 1988, p. 150). Surtout, la dernière génération de sables éoliens fossiles se met en place, entre Gafsa et les Grands Chotts (BALLAIS, BEN OUEZDOU, 1991). Il s'agit, au maximum, de $1 \mathrm{~m}$ de sables très quartzeux, fins à moyens, bien triés, $\left(98 \mu \mathrm{m}<\mathrm{Q}_{2}<174 \mu \mathrm{m}\right)$.

Ils ont subi ensuite une pédogenèse contemporaine de l'installation des sites néolithiques (cf. cidessous), tout comme les sables des Ziban (fig. 1) antérieurs à $6320 \pm 100$ B.P. (BALLAIS et al., 1979) dont ils sont très vraisemblablement contemporains. Au sud des Grands Chotts (El Hamma de Gabès, revers du Dahar = Monts de Matmata), des placages de sables limoneux éoliens, épais de quelques décimètres, présentent les mêmes caractéristiques.

\section{2 - L'HOLOCĖNE MOYEN (7 000 - 3500 B.P. ?): L'OPTIMUM CLIMATIQUE ?}

L'Holocène moyen se marque par la fin de l'accumulation de la basse terrasse et le développement de la pédogenèse et de l'hydrographie.

\subsection{La fin de l'accumulation de la basse terrasse}

D'une façon générale, la fin de l'accumulation de la basse terrasse est postérieure aux Capsiens et contemporaine des Néolithiques qui s'installent sur son sommet, par exemple à Bir Amir et à Aïn Edkouk (au sud de Tataouine), ou dans sa partie sommitale (oued Hallouf, au nord de Tataouine). Parfois, c'est encore un site épipaléolithique qui coiffe cette terrasse (oued Seradou, à l'ouest de Gabès ) ou un site capsien très tardif (6 $750 \pm 130$ B.P.) à la chebket Ouknina, au nord de Gabès (BALLAIS, 1973).

La série chronologique la plus complète, mais aussi la plus complexe, est celle de l'oued el Akarit (FONTES et al., 1983, ROGNON et al., 1983). Elle comporte une croûte gypseuse peu après $5995 \pm 50$ B.P., indication d'une phase de morphogenèse éolienne due à un assèchement (ROGNON et al., 1983). Cependant, l'accumulation se poursuit jusque vers $3680 \pm 160$ B.P., non sous forme de loess (idem), mais de limons fluviatiles, en même temps que les débits de l'oued augmentent à partir de 3910 B.P., (ZOUARI, 1988, p.150). Plus au sud-est, une terrasse de l'oued Mdou est datée de $5195 \pm 105$ B.P. (STEINMANN, BARTELS, 1982). Dans l'Extrême-Sud, des dépôts de cette terrasse s'accumulent encore vers $6580 \pm 350$ B.P. (PETIT-MAIRE et al., 1991).

\subsection{L'épanouissement de la pédogenèse}

Sur les sables éoliens qui se sont fixés progressivement, les populations néolithiques s'installent et la pédogenèse se développe, y compris à l'intérieur du Grand Erg Oriental comme à l'ouest de Nefta. 
Par exemple, sur le piémont nord du djebel Morra, les $20 \mathrm{~cm}$ supérieurs se caractérisent par une structure grumeleuse très développée, probablement due à la bioturbation par des vers de terre. Ils présentent de fort taux d' $\mathrm{Al}_{2} \mathrm{O}_{3}(3 \%)$ et de $\mathrm{Fe}_{2} \mathrm{O}_{3}(1,4 \%)$ qui confirment cette pédogenèse. En dessous, les taux diminuent mais une structure prismatique apparaît, le gypse devient plus abondant (6\%) et s'individualise sous forme de fines tubulures de racines. Localement, des nodules calcaires sont abondants. Très comparables aux sols contemporains des Ziban (BALLAIS et al., 1989), ils se sont formés dans l'étage aride du climat méditerranéen, alors qu'ils occupent actuellement l'étage saharien.

Les gisements néolithiques associés sont datés de $5858 \pm 66$ B.P. et $5930 \pm 87$ B.P. (BALLAIS, BEN OUEZDOU, 1991). Les populations consomment alors de préférence Helix melanostoma, aujourd'hui réfugié plus au nord. Au-delà de la frontière algérienne, les paléosols à Helicella de l'erg El Oussif (fig.1), un peu plus au nord, ont un âge comparable: $5930 \pm 1780$ B.P.

Plus au sud, et sur substrat très gypseux, en particulier au sommet de la terrasse holocène, des sols se développent formant aujourd'hui des croûtes gypseuses dures, par exemple le long de l'oued Bir Amir ou le long de l'oued Jenain (PETIT-MAIRE et al., 1991). Ils sont caractéristiques de l'étage saharien et indiquent que, à l'Holocène moyen, la zonation actuelle existe, mais décalée vers le sud.

A la différence des écoulements générateurs de la basse terrasse et du glacis, ces sols supposent des géosystèmes en équilibre, une longue stabilité de la surface topographique permettant le développement d'une végétation. Donc une répartition saisonnière des précipitations meilleure que lors de l'Holocène inférieur, accompagnée d'une intensité plus faible (fig. 2).

\subsection{Les preuves d'une hydrographie abondante}

L'accumulation de la terrasse ne résulte pas d'une simple augmentation de la charge solide des oueds. La sédimentologie de cette terrasse montre que le régime des oueds s'est amélioré. Les traces d'hydromorphie se multiplient: pseudo-gley et accumulations de $\mathrm{MnO}_{2}$ (oued Hallouf), fin dépôt aquatique de gypse bien lité (oued Bire Amir, oued el Akarit). Localement, des mares peuvent apparaître, à l'oued el Akarit jusque vers $5995 \pm 500$ B.P. ou encore dans l'oued Bou Zayane, à l'ouest de Gafsa (BEN OUEZDOU, sous presse). Plus généralement, les débits sont plus soutenus, plus réguliers: l'oued Jenain (fig. 3), interrompu actuellement par un bouchon dunaire à l'aval du borj Jenain, coulait jusqu'au Grand Erg Oriental et les oueds Abdallah et Makhrerouga construisaient un travertin peut-être vers $6970 \pm 70$ B.P. (PETIT-MAIRE et al., 1991).

Enfin, c'est très probablement à cette époque que les aïoun (= sources), aujourd'hui fossiles dans la partie méridionale du chott Rharsa, au nord du chott Jerid, fonctionnent pour la dernière fois. En effet, ils sont parfois accompagnés de placages sableux éoliens pédogénétisés et d'industries néolithiques à Leucochroa candidissima tout à fait comparables à ceux des dunes proches.

\section{3 - L'HOLOCÉNE SUPÉRIEUR PRÉHISTORIQUE: L'ASSĖCHEMENT CLIMATIQUE PROGRESSIF}

Cette période est très mal connue, pour deux raisons. La première est la rareté des dépôts ou des formes qui viennent s'interposer entre la basse terrasse (ou les sols) de l'Holocène moyen et la très basse terrasse. La seconde, partiellement liée à la précédente, est l'absence à peu près totale de datations sur cette période, aussi bien datations isotopiques qu'archéologiques, en raison de l'inexistence locale de Chalcolithique ou d'Âge du Cuivre ou du Bronze.

\subsection{L'entallie de la basse terrasse}

Ce qui semble certain, c'est le changement progressif de la morphogenèse fluviale: peu à peu, et dans tous les oueds, la basse terrasse est entaillée à la vitesse moyenne de 1,2 mm/an (BALLAIS, $1991 \mathrm{~b}$ ). Très probablement, ce renversement correspond à une aridification du climat (BRUN, 1989) (fig. 2). 


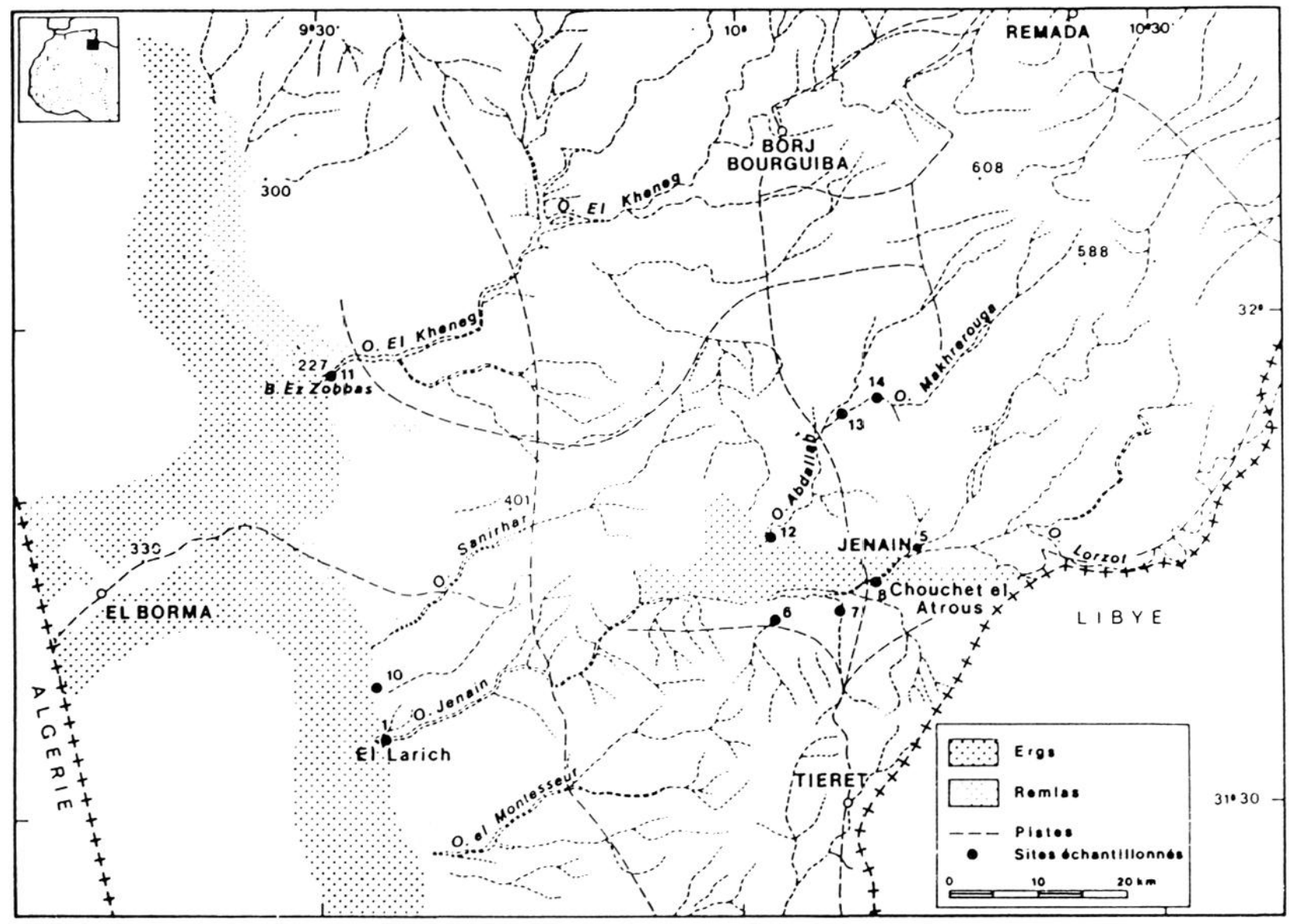

Fig. 3 - SITES DE L'EXTRÊME-SUD TUNISIEN.

\subsection{Le développement de la déflation}

L'aridification du climat reste insuffisante pour permettre la remobilisation des sables par le vent: l'aridité considérable du Pléniglaciaire (BALLAIS, HEDDOUCHE, 1991) ne sera jamais atteinte. Cependant, dans le chott Rharsa, les fonds de sebkha attribués à l'Holocène moyen sont maintenant en inversion de relief, $1,5 \mathrm{~m}$ à $2 \mathrm{~m}$ au-dessus du fond actuel. Il s'agit d'ailleurs d'un secteur limité, où la déflation et la corrasion restent très efficaces aujourd'hui, car, ailleurs, l'ablation par le vent est très réduite (COQUE, 1962).

\subsection{Les incertitudes chronologlques}

Le début de l'aridification est traditionnellement placé vers 4 500-4 000 B.P. (BALLAIS et al., 1979). Cependant, l'oued el Akarit présenterait encore de forts débits jusque vers 3000 B.P. (ZOUARI, 1988, p. 150), en accord avec la construction prolongée de terrasses plus au nord, dans l'étage aride ou semi-aride (LUBELL et al., 1976). A l'inverse, dans l'Extrême-Sud, les plus récents épandages à Helicella sp. de la Graret Abdallah (fig. 3, site 12) s'arrêtent vers $5640 \pm 100$ B.P. (PETIT-MAIRE et al., 1991). La zonation actuelle paraît ainsi acquise, à partir de la fin de l'Atlantique européen (fig. 2).

\section{4 - L'HOLOCĖNE SUPÉRIEUR HISTORIQUE}

\subsection{Un éplsode humide vers 2400 B.P. ?}

A Ksar Rhilane, au sud-ouest des Monts de Matmata, au milieu des premières barkhanes du Grand Erg Oriental, se sont déposés quelques décimètres de sables, limons et argiles à litage fluviatile, très riches en $\mathrm{Al}_{2} \mathrm{O}_{3}$ et $\mathrm{Fe}_{2} \mathrm{O}_{3}$. Cet épisode a été suffisamment long pour que des Helicella (datées $2380 \pm 155$ B.P.), qui vivent aujourd'hui à plusieurs dizaines de kilomètres plus au nord, viennent s'installer sur les Graminées qui poussent alors. Il faut donc envisager plus qu'un évènement exceptionnel, une augmentation considérable 
des ruissellements qui n'atteignent plus, aujourd'hui, Ksar Rhilane, en liaison évidente avec une pulsation climatique humide. Mais cet épisode n'a pas encore été identifié systématiquement ailleurs en bordure nord du Sahara, même si des indices plus ou moins contemporains existent dans l'Atlas saharien (BALLAIS, 1976).

\subsection{L'accumulation de la très basse terrasse historique}

La terrasse historique est moins développée dans le Sud tunisien et même en Libye (BALLAIS, 1991b) que dans le centre et le nord de la Tunisie (BALLAIS, 1991a). Très souvent, l'entaille précédente a continué, dans la terrasse holocène, sans stade d'arrêt ou de remblaiement, particulièrement le long des oueds secondaires.

Par contre, le long des oueds plus importants, une très basse terrasse s'emboîte ou s'étage dans la précédente. Dans ce cas, la base est souvent grossière et la partie sommitale plus fine, sableuse en général. Son âge historique est prouvé par la présence de tessons de céramique romaine (BALLAIS et al., 1988, BALLAIS, 1991a). Localement, sur l'oued Seradou, elle commence à s'accumuler au $\mathrm{II}^{\circ}$ ou au $\mathrm{III}^{\circ} \mathrm{s}$. de notre ère (BALLAIS, 1990). Elle disparaît dans l'Extrême-Sud.

Une fois de plus, l'oued el Akarit se signale par la richesse et la complexité de son comportement. Deux terrasses historiques y coexistent: la plus haute, datée $1470 \pm 190$ B.P. (PAGE, 1972) et la plus basse, datée $610 \pm 110$ B.P. (FONTES et al., 1983).

Le problème de la genèse de cette terrasse historique, très générale autour de la Méditerranée (VITAFINZI, 1969), se pose en termes radicalement différents de celui des formes plus anciennes car il est nécessaire d'envisager le rôle des facteurs anthropiques (en particulier, l'extension et les modalités de l'occupation du sol). Cette nécessité est renforcée par l'importance de la vitesse moyenne d'accumulation de cette terrasse: $7,4 \mathrm{~mm} / \mathrm{an}$, soit environ 5 fois plus rapidement que la terrasse holocène (BALLAIS, 1991b) !

\subsection{L'entallie de la très basse terrasse et la remobilisation des sables collens}

Après cet épisode d'accumulation, l'entaille verticale généralisée reprend à la vitesse moyenne minimum considérable de 3,2 mm/an (BALLAIS, 1991b). A la différence des cours d'eau plus septentrionaux, il est exceptionnel qu'une ou deux banquettes s'y individualisent (BALLAIS et al., 1988).

Depuis peu, et de plus en plus, les sables des dunes et versants aspergés fixés sont remobilisés, avec une intensité jamais atteinte depuis le Pléniglaciaire. Ce mouvement qui date parfois de moins de 20 ans est, certes, lié à l'aridification postérieure à l'optimum holocène. Mais, surtout, il est dû à la désertification qui progresse à partir des champs labourés, des puits et des villages, y compris là où les précipitations moyennes annuelles dépassent $100 \mathrm{~mm} / \mathrm{an}$.

\section{CONCLUSIONS}

Il apparaît nettement que la phase humide de l'Holocène inférieur est plus précoce que ne l'indique certaine synthèse récente (ROGNON, 1987) et que l'aridité tend à disparaître dès le Tardiglaciaire (FONTES, GASSE, 1989). De ce point de vue, la chronologie se rapproche considérablement de celle utilisée pour le Sahara tropical (fig. 2). Dans le même sens, la petite phase aride vers $7500-7000$ B.P. (?) rappelle celle mise en évidence depuis longtemps dans le bassin du Tchad (SERVANT, 1973).

Par contre, pour ce qui est de l'importance de la phase humide postérieure à 7000 B.P., c'est plutôt vers l'Atlantique de la chronologie européenne qu'il faut se tourner. Dans le même sens, l'accumulation de la basse terrasse holocène semble bien, pour l'essentiel, contemporaine du «remblaiement holocène principal» des Alpes du Sud (JORDA, 1985).

Pour l'Holocène supérieur préhistorique, la chronologie saharienne tropicale devient évanescente, en raison de l'accroissement de l'aridité et le petit nombre de témoins tunisiens interdit encore une comparaison systématique avec la chronologie européenne. Pourtant, c'est sûrement dans cette voie que les progrès sont à espérer (BALLAIS, 1976) à condition de tenir compte des spécificités méditerranéennes comme nous y incite l'existence d'au moins une terrasse historique.

Finalement, le Sud tunisien est l'un des espaces privilégiés qui devraient permettre d'articuler les chronologies post-glaciaires de la zone tempérée et de la zone tropicale sur le fuseau eurafricain. 
Note: sauf indication contraire, les industries préhistoriques inédites ont été déterminées par A. GRAGUEB que je remercie très vivement.

\section{RÉFÉRENCES BIBLIOGRAPHIQUES}

BALlAIS J.-L., (1973). - Données nouvelles sur le Pléistocène récent de la Tunisie méridionale, Bull. Soc. Hist. Nat. Afr. nord, Alger, t. 64, fasc. 3-4, p. 129-150.

BALLAIS J.-L., (1976). - Morphogenèse holocène dans la région de Chéria (Nementchas-Algérie), Actes Symp. Versants en Pays méditer., Aix-en-Provence, CEGERM, vol. V, p. 127-130.

BALLAIS J.-L., (1990). - Terrasses de culture et jessours du Maghreb oriental, Méditerranée, Aix-en-Provence, T. 71, 3.4 , p. $51-53$.

BALlAIS J.-L., (1991a). - Les terrasses historiques de Tunisie, Z für Geom., Berlin, Suppl. Band 83, p. 221-226.

BALLAIS J.-L., (1991b). - Vitesses d'accumulation et d'entaille des terrasses alluviales holocènes et historiques au Maghreb oriental, Physio-Géo, Paris, N²2-23, p. 89-94.

BALLAIS J.-L., MARRE A., ROGNON P., (1979). - Périodes arides du Quaternaire récent et déplacement des sables Éliens dans les Ziban (Algérie), Rev. Géogr. phys. et Géol. dyn., Paris, vol. 21, fasc. 2, p. 97-108.

BALLAIS J.-L., BOURGOU M., KARRAY R., LAUTRIDOU J.-P., LEVANT M., OUESLATI A., BEN OUEZDOU H., BOUHAFA T., GRAGUEB A., (1988). - Premiers résultats du programme d'étude de l'Holocène de Tunisie, Méditerranée, Aix-en-Provence, $n^{\circ} 2$, p. 64-67.

BALLAIS J.-L., DUMONT J.-L., LE COUSTUMER M.-N., LEVANT M., (1989). - Sédimentation éolienne, pédogenèse et ruissellement au Pléistocène supérieur-Holocène dans les Ziban (Algérie), Rev. Géom. dynam., Paris, $\mathrm{n}^{\circ}$ 2, $\mathrm{p}$. 49-58.

BALLAIS J.-L., BEN OUEZDOU H., (1991). - Sables éoliens quaternaires entre les chaînes de Gafsa et du Cherb (Sud tunisien), Z. für Geom., Berlin, suppl. Bd. 83, p. 221-226.

BALLAIS J.-L., HEDDOUCHE A., (1991). - Bas Sahara septentrional et Grand Erg Oriental, Coll. PICG 252 : Paléomilieux et peuplements préhistoriques sahariens au Pléistocène supérieur, Solignac, 23 p.

BRUN A., (1989). - Microflores et paléovégétations en Afrique du Nord depuis 30.000 ans, Bull. Soc. géol. Fr., Paris, $(8)$, t. $\mathrm{V}, \mathrm{n}^{\circ} 1$, p. $25-33$.

COQUE R., (1962). - La Tunisie présaharienne. Etude géomorphologique, A. Colin, Paris, 476 p.

FONTES J.-C., COQUE R., DEVER L., FILLY A., MAMOU A., (1983). - Paléohydrologie isotopique de l'oued el Akarit (Sud Tunisie) au Pléistocène supérieur et à l'Holocène, Palaeogeogr., Palaeoclimatol., Palaeoecol., Amsterdam, 43, p. 41-62.

FONTES J.-C., GASSE F., (1989). - On the age of humid Holocene in late Pleistocene phases in North Africa; remarks on «Late Quaternary climatic reconstruction for the Maghreb (North Africa)» by P. Rognon, Palaeogeogr., Palaeoclimatol., Palaeoecol., Amsterdam, 70, p. 393-398.

GRAGUEB A., (1983). - Ibéromaurusien et industries à lamelles en Tunisie, Thèse $3^{\circ}$ cycle, Aix-Marseille I, 2 vol., 229 p., ronéot.

HARBI-RIAHI M., (1989). - Le site préhistorique de Bir Oum Ali, Excursion Guidebook, PICG 252, Djerba, p. $46-47$.

JORDA M., (1985). - La torrentialité holocène des Alpes Françaises du Sud. Facteurs naturels et facteurs anthropiques de son évolution, Coll. INQUA, Toulouse, 1983, Cahiers Ligures de Préh. et de Protoh., Carcassonne, N.S., $\mathrm{n}^{\circ}$ 2, p.49-70.

LUBELL D., HASSAN F.A., GAUTIER A., BALLAIS J.-L., (1976). - The Capsian Escargotières, Science, Washington, vol. $191, \mathrm{n}^{\circ} 4230$, p. $910-919$.

PAGE W.D., (1972). - The geological setting of the archaeological site at oued el Akarit and the palaeoclimatic significance of gypsum soils (S.-Tunisian), PhD Thesis, Univ. of Colorado, 111 p., ronéot.

PETIT-MAIRE N., DELIBRIAS G., (1979). - Late Holocene palaeoenvironment in the Ghadames area, The Maghreb Review, London, 4/4-6, p. 138-139.

PETIT-MAIRE N., BUROLLET P.F., BALLAIS J.-L., FONTUGNE M., ROSSO J.-C., LAZAAR A., (1991). - Paléclimats holocènes du Sahara septentrional. Dépôts lacustres et terrasses alluviales en bordure du Grand Erg Oriental à l'extrême-sud de la Tunisie, C.R. Acad. Sci. Paris, t. 312, série II, p. 1661-1666.

ROGNON P., (1987). - Late Quaternary climatic reconstruction for the Maghreb (North Africa), Paleogeogr., Palaeoclimatol., Palaeoecol., Amsterdam, 58, p. 11-34.

ROGNON P., LEVY A., BALLAIS J.-L., COUDÉ G., RISER J., (1983). - Essai d'interprétation des coupes du Quaternaire récent de l'oued el Akarit (Sud tunisien), Géol. Méditer., Marseille, t. X, nº 2, p. 71-91.

SERVANT M., (1973). - Séquences continentales et variations climatiques: évolution du bassin du Tchad au Cénozoïque supérieur, Thèse ès-Sciences, Paris, 348 p., ronéot.

STEINMANN S., BARTELS G.K., (1982). - Quartärgeomorphologische Beobachtungen ans Nord und Sud Tunesien, Catena, Cremlingen, vol. 9, p. 95-108.

VITA-FINZI C., (1969). - The Mediterranean Valleys. Geological Changes in Historical Times, Cambridge Univ. Press, $140 \mathrm{p}$.

ZOUARI K., (1988). - Géochimie et sédimentologie des dépôts continentaux d'origine aquatique du Quaternaire supérieur du Sud tunisien: interprétations paléohydrologiques et paléoclimatiques, Thèse ès-Sciences, Paris-Sud, $321 \mathrm{p}$. ronéot. 